\title{
Gesprächsanalytische Rekonstruktion von mündli- chen Verhandlungen im Gericht und in alternativen Institutionen des Rechts 1
}

\section{Einleitung mit einigen Anmerkungen zur Forschungslage}

Nach wie vor ist die mündliche Verhandlung vor Gericht nur im Ansatz zum Gegenstand von genuin gesprächsanalytischen Rekonstruktionen gemacht worden, also von ethnomethodologisch orientierten Analysen auf der Basis von Tonaufnahmen authentischer Verhandlungen und deren Verschriftlichung. Diese Aussage bezieht sich natürlich ausschließlich auf den deutschsprachigen Raum. Ausnahmen sind z.B. Hoffmann 1980a und 1980b, Caesar-Wolf \& Breunung 1981, Ullmer-Ehrich 1981, Hoffmann 1983, Rehbein 1989 und Schröder 1995.² Die einzigen umfassenderen Studien, die auf der Analyse von Tonaufnahmen und Transkriptionen basieren, waren bisher Hoffmann 1983 und Schröder 1995. Dabei geht Hoffmann eher von pragmatisch-sprechakttheoretischen Beschreibungsansätzen aus (Beschreibung von Diskurstypen, Sprechaktsequenzen und Sprechhandlungsmustern), und nur Schröder formuliert als Erkenntnisinteresse die gesprächsanalytische Rekonstruktion der komplexen kommunikativen Wirklichkeit von Verhandlungen vor Gericht.

1 Der Beitrag ist weder als Einführung in gesprächsanalytische Arbeitsweise gedacht noch als Übersicht über gesprächsanalytische Untersuchungen von Gerichtsverhandlungen; vielmehr stellt er eine etwas ausführlichere Nachbereitung meiner Diskussionsbeiträge während des Workshops "Legal Texts" im Rahmen des LSP-Symposiums in Kopenhagen 1997 dar. - Von Ulrich Reitemeier (Institut für deutsche Sprache Mannheim) bekam ich wertvolle Hinweise für die Abfassung dieses kleinen Artikels; Ulrich Heuberger (Høgskolen i Østfold/Halden) möchte ich für das Korrekturlesen danken.

2 Die Literatur zur Erforschung der juristischen Kommunikation insgesamt bis einschließlich 1985 ist dokumentiert und zum größten Teil ausführlich kommentiert in Reitemeier 1985.

* Peter Schröder

Høgskolen i Østfold

Os Allé 9

$N$-1757 Halden 
Diesen Einleitungsabsatz muß ich nun gleich wieder etwas einschränken: Während ich diesen kleinen Artikel abfaßte, stieß ich auf die 'konversationsanalytische Untersuchung zur Glaubwürdigkeit in Strafverfahren' von Stephan Wolff und Hermann Müller (Wolff \& Müller 1997); bei Wolff \& Müller 1997: 9 findet sich dann auch in der "Danksagung" eine ebenso feinsinnige wie überzeugende Skizzierung der Chancen und Vorteile einer ethnomethodologisch orientierten Einstellung zum Forschungsgegenstand und zur eigenen Analysepraxis:

"Es gehört zur Programmatik qualitativer Forschung, sich ganz bewußt der Eigensinnigkeit des Untersuchungsfeldes auszusetzen. Das bedeutet vor allem, aus freien Stücken auf jenen Schutzschild gegen die Unordentlichkeit der Welt zu verzichten, welcher die Methodenlehre in Gestalt von Experimentalplänen, statistischen Modellen, wohlformulierten Fragebögen u.ä. in so reichem Maße bereithält. Es gilt, der Versuchung zu widerstehen, sich die Daten schon bei deren Erhebung forschungs- bzw. theoriegerecht zurechtzustutzen. Das soziale Geschehen sollte nicht vorschnell interpretierend rekonstruiert, sondern zunächst einmal bloß registriert und in seiner Komplexität und natürlichen Prozeßhaftigkeit soweit möglich konserviert werden. Zudem sehen sich qualitative Forscher mit dem Anspruch konfrontiert, etwaige Ordnungsstrukturen aus dem Material herauszudestillieren und nicht schon vorgedachte und aus anderen Zusammenhängen mitgebrachte Ordnungskonzepte bloß mit diesem zu konfrontieren (oder gar ihre Daten zu bloßen Illustrationszwecken zu mißbrau(hen)."

Bezieht man die alternativen Institutionen zur Regelung von Rechtsfällen mit ein, so sind an dieser Stelle ebenfalls die Arbeiten zu nennen, die im Zusammenhang mit dem Projekt "Schlichtung - Gesprächs- und Interaktionsanalyse eines Verfahrens zur Bewältigung sozialer Konflikte" entstanden sind, ${ }^{3}$ und zwar diejenigen, die sich mit der gütlichen Beilegung von Nachbarschaftsstreitigkeiten in der Vergleichsbehörde oder vor dem Schiedsmann beschäftigen. ${ }^{4}$ In diesem Rahmen muß auch die Arbeit von Deppermann 1997 zum interaktiven Umgang mit Glaubwürdigkeit in Schlichtungs- und Streitgesprächen genannt werden, der

3 An dem Projekt waren beteiligt Wolfgang Klein, Werner Nothdurft (Leitung/Koordination), Ulrich Reitemeier und Peter Schröder; das Projekt wurde von 1983 bis Ende 1989 am Institut für deutsche Sprache in Mannheim mit Unterstützung der DFG durchgeführt.

4 Nothdurft 1986, Spranz-Fogasy 1986, Klein 1987, Klein/Nothdurft 1987, Nothdurft 1987, Reitemeier 1987, Schröder 1987, Nothdurft 1989, Klein 1995, Nothdurft 1997 und Schröder/Klein/Nothdurft/Reitemeier 1997. 
auch mit Materialien aus dem Mannheimer Schlichtungsprojekt arbeitet.

Daß trotz aller Verdienste der genannten Arbeiten die mündliche Gerichtsverhandlung, um die es mir hier vor allem geht, bisher nur im Ansatz empirisch wirklich erforscht ist, hat natürlich Gründe: Als wir im obengenannten Schlichtungsprojekt in mühseliger und anstrengender Überzeugungsarbeit schließlich Zugang zu verschiedenen Gerichten gefunden hatten, d.h. die Genehmigung, die jeweilige Schlichtungspraxis auf Tonband aufzunehmen, erschien gleichzeitig eine Studie zur Arbeitsgerichtsbarkeit, die genau an diesem Punkt passen mußte (Rottleuthner 1984). In der Anfangsphase dieses Projekts hatte Rottleuthner entsprechend darüber geklagt, daß über die konkrete kommunikative Realität, über konkrete interaktive Zusammenhänge in Verhandlungen vor dem Arbeitsgericht keine oder nur sehr ungenaue Aussagen möglich wären, weil das Problem von Tonaufnahmen mündlicher Verhandlungen nicht gelöst sei (vgl. Rottleuthner 1978: 116). Und in der Tat: Es gilt für Gerichte wie für alle anderen Institutionen, die sich als hermetisch verstehen, wie etwa auch Wirtschaftsunternehmen: Die Bedenken, die eigene kommunikative Praxis analytischer Rekonstruktion zugänglich zu machen, sind groß und stellen für den Empiriker, ob er nun von der Rechtssoziologie, der Soziologie oder der Linguistik kommt, oft unüberwindbar scheinende Barrieren dar. Der Weg führt in der Regel nur über langfristig angelegte soziale Kontakte, die das nötige Vertrauen schaffen. 5

Die Zugangsprobleme sind unverändert groß - trotz der Untersuchung, die jetzt von Wolff \& Müller vorgelegt wurde, und auch wenn es im Rahmen des Schlichtungsprojekts gelungen ist, ein relativ großes Korpus von Tonaufnahmen von Güteverhandlungen vor dem Arbeitsgericht (ca. 80 Verhandlungen) aufzubauen, zusätzlich zu einem kleineren Korpus mit Sozialgerichtsverhandlungen, einem Korpus mit 20 Verhandlungen aus sechs Zivilsenaten eines Oberlandesgerichts und einem Korpus mit einigen Amts- und Landgerichtsverhandlungen. Sieht man einmal von Schröder 1995 ab, so sind diese Verhandlungen bisher

\footnotetext{
5 Meine eigenen Erfahrungen mit dem empirischen Zugang zu Gerichtsverhandlungen und dem Zugang zu authentischer mündlicher Wirtschaftskommunikation bestätigen das. Allerdings gibt es auch den nicht seltenen Fall, daß über private Zufälligkeiten der langwierige Prozess der Vertrauensherstellung erheblich abgekürzt wird.
} 
leider nicht systematisch gesprächsanalytisch bearbeitet worden. ${ }^{6}$ Es ist kaum verständlich, daß das Institut für deutsche Sprache dieses einmalige Material, das in zäher und aufwendiger 'Akquisitionsarbeit' zusammengetragen wurde, nicht zum Gegenstand gezielter gesprächsanalytischer Rekonstruktion macht, ganz zu schweigen davon, daß dadurch die Richter und Anwälte, die ihre Zustimmung zu den Aufnahmen gegeben hatten, in ihren Erwartungen, durch solche Analysen in der kritischen Reflexion ihrer eigenen kommunikativen Praxis angeregt und unterstützt zu werden, schwer enttäuscht wurden. Ein Grund für dieses Versäumnis ist sicherlich auch, daß nach wie vor für die vorherrschende Linguistik in der Bundesrepublik die gesprächsanalytische Rekonstruktion der kommunikativen Wirklichkeit in konkreten gesellschaftlichen Institutionen nicht zu den genuin linguistischen Erkenntnisinteressen gehört.

Was Gesprächsanalyse kann, ${ }^{7}$ ist überzeugend eigentlich nur durch ihren Vollzug zu demonstrieren; das würde für den Fall dieses kleinen Artikels bedeuten, daß ich einen Ausschnitt aus einer Gerichtsverhandlung zunächst 'turn - by - turn', also streng sequentiell, rekonstruieren müßte, indem ich die einzelnen 'turns' und 'turn'-Konstruktionseinheiten danach kategorisiere, welche interaktiven Aufgaben jeweils wahrgenommen werden, ob auf der Ebene der Verhandlungs- oder Gesprächsorganisation, der Ebene der Herstellung sozialer Beziehungen und Identitäten, der Sachverhaltsebene, der Ebene der Handlungskonstitution und -koordination, auf der Ebene der Verständigungssicherung oder der Ebene der Interaktionsmodalitäten, ${ }^{8}$ nachzeichne, in welcher Weise die Beteiligten jeweils auf mehreren Ebenen der Gesprächskonstitution gleichzeitig etwas tun, rekonstruiere, wie sich die Teilnehmer

6 Im Projekt Gesprächsrhetorik des Instituts für deutsche Sprache Mannheim (Projektleiter: Werner Kallmeyer) bilden diese Korpora von Gerichtsverhandlungen einen Teil der empirischen Basis; Gegenstand des Projekts sind kommunikative Verfahren der Konflikt- und Problembearbeitung; vgl. dazu Kallmeyer 1996. Außerdem arbeitet Otmar Bettscheider (Mannheim) an einer Promotion, die sich mit Aspekten der Handlungskoordination zwischen Professionellen und Laien in den Oberlandesgerichtsverhandlungen auseinandersetzt.

7 Zur Einführung in die Gesprächs- bzw. Konversationsanalyse (die Diskussion über Gemeinsamkeiten und Unterschiede will ich hier nicht aufnehmen) vgl. vor allem Bergmann 1981 und 1988; siehe auch Henne/Rehbock 1979 und Brinker/Sager 1989.

$8 \mathrm{Zu}$ den Konstitutionsebenen im Gespräch vgl. Kallmeyer/Schütze 1976. 
ihre Aktivitäten wechselseitig zu verdeutlichen versuchen, zeige, wie interaktiv mit dem Phänomen der 'konditionellen Relevanz' umgegangen wird usw., aufdecke, welche interaktiven Muster die Beteiligten realisieren, systematisch Handlungsorientierungen und Gesprächsstrategien aufdecke, latente Kommunikationsprobleme oder -konflikte offenlege, die Ursachen von Turbulenzen oder Konflikten im Gespräch aufspüre und völlig oder partiell mißlungene Handlungskoordination zwischen den Beteiligten verdeutliche. Dafür aber reicht hier der Raum nicht aus; allein der notwendige Abdruck von Transkriptpassagen würde ihn sprengen. Und so bleibt mir die undankbare Aufgabe, die Möglichkeiten von Gesprächsanalyse, sieht man von der Präsentation und andeutungsweisen Analyse dreier kleiner Gesprächsausschnitte ab, quasi von außen beschreiben zu müssen.

\section{Einige Aspekte der kommunikativen Wirklichkeit von Gerichtsverhandlungen, die nur über Gesprächsanalyse zugänglich sind}

\subsection{Die interaktive Herstellung von Sachverhalten und das Problem der Handlungskoordination zwischen den Beteiligten}

Die Chancen und Möglichkeiten, die sich über gesprächsanalytische Rekonstruktionen von Gerichtskommunikation für eine kritische Reflexion dieser gesellschaftlichen Praxis eröffnen, möchte ich zunächst etwas beleuchten im Zusammenhang mit Sachverhalten und Konfliktversionen. Sachverhalte, Streitgegenstände oder Tatbestände "existieren" in einer Verhandlung im wesentlichen als symbolische Repräsentationen, also in sprachlichen Darstellungen. In einer Verhandlung, also indem mit den strittigen oder fraglichen Sachverhalten umgegangen wird, werden diese Sachverhalte und die kontroversen Verhandlungspositionen jeweils immer wieder neu hergestellt, ob in den einleitenden auf Grund der Aktenlage und der eingegangenen Schriftsätze schriftlich vorbereiteten Sachverhaltsrekonstruktionen des Gerichts oder ob in den Anschuldigungen und Stellungnahmen der Parteien und Parteienvertreter. In diesen Sachverhaltsdarstellungen und den Aushandlungs-, Durchsetzungs- und Überprüfungsaktivitäten werden die Sachverhalte auch nach Maßgabe der jeweiligen Handlungsorientierungen, der je- 


\section{8}

weiligen Konfliktsicht der Beteiligten, umstrukturiert, umdefiniert, durch unterschiedliche Relevanzsetzung einzelner Sachverhalts- oder Konfliktbestandteile verändert, kurz: der Verhandlungsgegenstand ist Gegenstand bzw. das Produkt ständig neuer interaktiver Herstellungsleistungen der Beteiligten. Die Prozesse selbst und die komplizierte interaktive Dynamik, die von diesen in der Verhandlung von den Beteiligten erbrachten Konstitutionsleistungen ausgeht, kann nur eine gesprächsanalytische Rekonstruktion mit Transkript und Aufnahme wirklich aufdecken. Dem Beobachter mit Stift und Papier entzieht sich diese Dynamik, bzw. er wird nur in der Lage sein, zufällige Teilaspekte dieser komplexen interaktiven Zusammenhänge quasi ad hoc festzuhalten, und der Erkenntniswert solcher 'ad hoc'-Zuschreibungen von analytischen Kategorien zu simultan ablaufendem interaktivem Geschehen ist zweifelhaft, da sie aufgrund der Flüchtigkeit des Gesprochenen nicht überprüfbar sind. Der Gesprächsanalytiker dagegen kann das Band anhalten und es beliebig oft zurückspulen, und er kann mit Hilfe des Transkripts seine eigene Wahrnehmung des Geschehens beliebig verlangsamen. Auf diese Weise kommt er mit seinen Analyseperspektiven 'Rekonstruktion der Verhandlungsbeiträge aus der Perspektive der Beteiligten', 'Zuschreibung einer Kategorie zu einzelnen 'turns' oder 'turn'-Konstruktionseinheiten, die die wahrgenommene interaktive Aufgabe abbildet', 'systematische Aufdeckung von Handlungsorientierungen der Beteiligten', 'Aufzeigen, in welcher Weise die Aktivitäten der Beteiligten 'recipient designed' sind', 'Rekonstruieren, mit welchem Verständnis der Aktivitäten der anderen Beteiligten ein Beteiligter umgeht' oder 'Rekonstruktion von Mustern für die Sachverhaltsrekonstruktion oder -darstellung' den Vorgängen der Herstellung von sozialer Bedeutung wirklich auf die Spur.

Ich möchte das theoretisch Erläuterte in seiner Relevanz zumindest durch die Schilderung eines Falls etwas verdeutlichen: In einer Verhandlung vor dem Sozialgericht (3003/31: "Abgebrochene Maßnahme") klagt die Klägerin (Aussiedlerin aus einem kommunistischen Staat) gegen erhebliche Rückforderungen des Arbeitsamtes für - so das Arbeitsamt - Überzahlungen für eine sog. Maßnahme (Deutschunterricht für den Sohn an einer privaten Sprachschule). Der Sohn war krank geworden, hatte faktisch die 'Maßnahme' abgebrochen, die Klägerin hatte aber, trotz starker eigener Bedenken, die sie auch der Schule gegenüber artikuliert hatte, auf mündliche Aufforderung der Schule hin 
(es bestünde ja schließlich ein Vertrag zwischen ihr und der Schule) weiterhin die vom Arbeitsamt bei ihr eingehenden Zahlungen für die 'Maßnahme' an die Schule weitergeleitet. Offizieller Verhandlungsgegenstand sind die Ansprüche des Arbeitsamtes gegenüber der Klägerin (die Maßnahme war faktisch abgebrochen, die Zahlungen hatten keine rechtliche Basis mehr), und dies ist auch der Sachverhalt, mit dem sich das Sozialgericht nur befassen kann und will. Die Forderungen und Ansprüche der Klägerin gegenüber der Schule sei kein Verhandlungsgegenstand, sondern diese müßte sie gegenüber der Sprachschule geltend machen. Die Klägerin hatte seinerzeit, als das Arbeitsamt eine solche Maßnahme für den Sohn initiiert hatte, noch im Arbeitsamt den Vertrag mit der Schule unterschrieben (das haben Arbeitsamt und private Schule offensichtlich der Einfachheit halber so geregelt). Die Klägerin hat eine andere subjektive Wahrnehmung der Situation: Aus einem kommunistischen Staat in die Bundesrepublik um- bzw. ausgesiedelt, ist sie mit privaten Ausbildungsverträgen nicht vertraut. In ihrer Wahrnehmung sind Arbeitsamt und Schule gesellschaftliche Institutionen, hinter denen sich die gleiche Instanz verbirgt, nämlich der Staat. Anders ist sie es nicht gewohnt. Entsprechend wäre es für ihr Rechtsempfinden logisch, daß die Schule das zu Unrecht empfangene Geld direkt ans Arbeitsamt zurückzahlt (sie ist empört über das Verhalten der Schule). Zusätzlich wird deutlich, daß sie in ihrem Selbstkonzept große Probleme mit der sozialen Rand- und Grenzsituation als Aussiedlerin hat. Ihre subjektive Sicht auf den Konfliktsachverhalt, ihre Konfliktorganisation, ist von großer Emotionalität und großem Leidensdruck geprägt. Die Verhandlung endet damit, daß der Richter die Klägerin des Gerichtsraumes verweist: frau below * darf ich sie bitten jetzt äh rauszugehn $\downarrow$ (Gespräch 3003/31: Abgebrochene Maßnahme). Die ca. 1stündige Verhandlung ist geprägt von mißglückter Handlungskoordination zwischen den Beteiligten; weder gelingt es der Klägerin, die professionelle Konfliktversion des Gerichts auch nur ansatzweise zu erfassen, noch schafft es der Richter, der subjektiven Konfliktsicht der Klägerin wirklich auf die Spur zu kommen. Seine Aufklärungs- und Beratungsanstrengungen greifen zu kurz. Allein diese knappe Falldarstellung dürfte ausreichen, um eine Vorstellung davon zu vermitteln, wie komplex und vielschichtig der turbulente Verlauf dieser Verhandlung ist, viel zu komplex und vielschichtig, um reiner Beobachtung zugänglich zu sein. 
Es hat sich in Gesprächen mit den Richtern im Sozial-, Arbeits- und Oberlandesgericht gezeigt, daß die Gesprächsanalyse hier insgesamt eine erhebliche Aufklärungsfunktion haben könnte und daß es sich z.B. anbieten würde, ein gesprächsanalytisch fundiertes Kommunikationstraining zum Programm von Richterfortbildungsseminaren zu machen oder - besser noch - in die Ausbildung von Juristen sowohl während des Studiums als auch während der Referendarzeit zu integrieren: Immer wieder glaubte ich bei Richtern ein - so meine ich - naives Sprachverständnis entdecken zu können, das davon ausgeht, daß sich hinter sprachlichen Formulationen und Mustern der 'reine' Sachverhalt, das Gemeinte, die eigentliche Sache, verbirgt, als sei jeweils nur ein wenig Anstrengung erforderlich, das Beiseiteräumen der störenden Formulierungen gleichsam, um zu diesem eigentlichen Sachverhalt vorzustoßen. Danach kann man dann - so dieses naive Sprachverständnis - mit der Sache in ihren objektiven Konturen umgehen und sie der üblichen juristischen Subsumtionspraxis unterwerfen usw.

Hier könnte der gesprächsanalytisch arbeitende Kommunikationstrainer oder Kommunikationsberater ${ }^{9}$ die Professionellen dafür sensibilisieren, was es heißt, daß Sachverhalte in Verhandlungen in der Regel nur als symbolische Repräsentationen existieren und daß sie jeweils interaktiv hergestellt werden müssen und welche interaktive Dynamik hiermit verbunden ist.

\subsection{Gesprächsanalyse und der Umgang mit Terminologie}

Ein Problem im Umgang mit Terminologie in Texten und mündlichen Situationen im Rahmen von "Fachsprachenforschung" 10 scheint mir zu sein, daß der Terminologiegebrauch in der Regel statisch beschrieben wird, d.h. in den Beschreibungen unberücksichtigt bleibt, in welchen funktionalen Kontexten Fachausdrücke und -formulierungen auftauchen, welche Rephrasierungs- und Übersetzungsaktivitäten folgen

\footnotetext{
9 Zu gesprächsanalytisch orientiertem Kommunikationstraining bzw. entsprechender Kommunikationsberatung für den Bereich 'Wirtschaftskommunikation' vgl. Brünner/ Fiehler 1998; ich könnte mir ähnliche Konzepte für juristische Kommunikation denken. $\mathrm{Zu}$ Gesprächsforschung und Kommunikationstraining vgl. auch Fiehlers Aufsatz im HSK-Band "Text- und Gesprächslinguistik" im 2. Halbband ("Gesprächslinguistik"); dieser Band ist in Vorbereitung.

10 Aus vielerlei Gründen ziehe ich es vor, von fachlicher Kommunikation zu sprechen; vgl. Schröder 1997.
} 
oder vorausgehen, wie sich Rephrasierungs- und Umschreibungs- bzw. Übersetzungsaktivitäten zu normativen Festlegungen verhalten, ob es spezifische Akzentuierungen oder vielleicht Ausblendungen definitorischer Merkmale gibt. Daß entsprechende analytische Perspektiven als Desiderat bezeichnet werden müssen, wurde m.E. auch während des Workshops "Legal Texts" deutlich. Ich will hier an einem kurzen Gesprächsausschnitt aus einer Güteverhandlung vor dem Schiedsmann demonstrieren, was ich mit einer solchen dynamischen gesprächsanalytischen Perspektive für die Beschäftigung mit Terminologieverwendung meine. Vor dem Schiedsmann oder in der Vergleichsbehörde werden vorgerichtlich alle Nachbarschaftsstreitigkeiten (außer Offizialdelikten) verhandelt, bevor ein Kläger (Antragsteller) - nach erfolglosem Einigungsversuch vor dem Schiedsmann - sich ans Amtsgericht wenden kann. In dieser Güteverhandlung geht es um eine Beleidigung, genauer darum, daß der Antragsgegner ('Beklagter') in einer alltäglichen Streitsituation gegenüber der Antragstellerin ('Klägerin') das sog. Götz-Zitat ${ }^{11}$ benutzt hat, also eine immer als ehrverletzend verstandene grobe Verbalinjurie. In der Phase der Konfliktrekonstruktion haben die Parteien jeweils aus ihrer Sicht den Konfliktsachverhalt etabliert, mit einer Fülle von Details und Facetten, die der jeweils anderen Partei den großen Teil der Schuld zuschreiben sollen. Der kleine Gesprächsausschnitt stellt einen Teil der Konfliktreformulierung aus der Sicht des Schlichters, des neutralen Dritten dar:

$01 \mathrm{C}$ \#herr < he"rrmann $\longrightarrow$ *\# bei allem was passiert is - *

$\mathrm{K}$ \#KLINGT WIE AUFRUF\#

C \#mach möglich sein — \# * aber- * dieser berühmte satz- den

K \#GENUSCHELT \#

C sie dort gegenüber frau blank— * haben laut werden lassen— *

$\mathrm{C} \quad$ das_ $—$ ist natürlich ne bel $\mid \_*$ ne beleidigung $\downarrow * *$ und beleidigungen

11 Gemeint ist damit die Verbalinjurie, die durch Goethes Sturm-und-Drang-Drama "Götz von Berlichingen" literaturfähig geworden ist und die in 'guten' Klassikerausgaben gewöhnlich durch Auslassung der Tabuwörter entschärft wird ("Er aber, sag's ihm, er kann mich - - "; Goethes Werke o.J.: Teil 3: 59); für den Leser, der jetzt noch stutzig ist: der Tabuausdruck lautet "am Arsche lecken”. Welche der im Gegenwartsdeutsch gängigen Varianten Herr Herrmann in der fraglichen Situation benutzt hat, ob ein "Leck mich!", "Du/Sie kannst/können mich mal!” oder etwa die deutlichere Version "Leck mich am Arsch!”, bleibt in der Verhandlung unerwähnt. 
05 C \#gehn ja— * werden ja nach dem strafgesetzbuch verfolcht— * \#

$\mathrm{K}$ \#PAPIERRASCHELN\#

C und zwar — * nach dem paragraph hundertfünfundachtzig bis

C hundertsiebenundachtzig a. und hundertneunundachtzig — * nach

C dem strafgesetzbuch $\downarrow *$

(Schröder (Hg.) 1997: 124) 12

Ohne daß ich nun diesen Ausschnitt in extenso analysieren will, folgende Verdeutlichungen zur Terminologieverwendung:

bei allem was passiert is— * mach möglich sein- : Der Schiedsmann blendet alle Konfliktbestandteile, die aus subjektiver Konfliktsicht des Antragsgegners Herrn Herrmann vielleicht relevant sind, in seiner Konfliktreformulierung aus professioneller Perspektive aus, markiert deutlich, daß diese anderen Aspekte des Sachverhalts aus seiner Perspektive nicht relevant sind.

* aber- * dieser berühmte satz- den sie dort gegenüber frau blank— * haben laut werden lassen— * : Der Schiedsmann markiert adversativ gegenüber den unwichtigen Bestandteilen des Konfliktsachverhalts den einen (aus seiner professionellen Perspektive) wichtigen, noch in einer alltagssprachlichen Umschreibung.

das— * ist natürlich ne bel/— * ne beleidigung $\downarrow$ ** : Der Schiedsmann subsumiert den einen Konfliktbestandteil, auf den er den komplexen Konfliktsachverhalt reduziert hat, unter die justitiable Kategorie 'Beleidigung', um dann unmittelbar anschließend (aus hier nicht wichtigen gesprächs- bzw. schlichtungsstrategischen Gründen) den zwangsläufigen Delikt-Strafe-Zusammenhang herzustellen (vgl. den Rest des Gesprächsausschnitts). ${ }^{13}$

Hier habe ich natürlich zu Demonstrationszwecken einen sehr einfachen Fall von professionell gesteuerter Sachverhaltsreduktion (das Ausblenden von 'unwichtigen' Konfliktbestandteilen), Terminologisierung des aus professioneller Sicht einzig relevanten Konfliktbestandteils und Subsumtion unter positives Recht vorgeführt. Ein solcher Pro-

12 Legende zu den benutzten Transkriptionszeichen siehe am Ende des Beitrags.

13 Vgl. die ausführliche Analyse dieser Verhandlung in Schröder/Klein/Nothdurft/ Reitemeier 1997; siehe auch Nothdurft 1997, der diese Analyse auch z.T. benutzt, ohne das allerdings zu markieren. 
zess kann sich über weit längere Verhandlungsphasen erstrecken und sich in überaus komplexen interaktiven Aushandlungsvorgängen zwischen den Beteiligten vollziehen, wobei dann oft nicht nur ein definitorisches Merkmal professioneller Terminologie involviert ist auf dem Wege zu der angestrebten terminologischen Reduktion des Sachverhalts. Entsprechend komplexe interaktive Vorgänge, die in ihrer Bedeutung erst sichtbar werden über eine sorgfältige und kleinschrittige Rekonstruktion ihrer Dynamik, können auch umgekehrt ablaufen: Die initiale Verwendung von professioneller Terminologie in Verhandlungen oder das selbstverständliche Voraussetzen, daß der Laie mit ihr vertraut ist, kann die Handlungskoordination zwischen Laien und Professionellen erschweren, kann umständliche 'Determinologisierungsaktivitäten', also Übersetzungs- und Verdeutlichungsanstrengungen, erforderlich machen, aber auch aufwendige Korrektur- und Reparaturanstrengungen bedingen angesichts von Turbulenzen oder Blockaden in der Verhandlung, die ihren Ursprung in kontraproduktiver Verwendung professioneller Terminologie hatten. Einen eklatanten Fall von Wahrnehmungsdivergenzen zwischen Professionellen und Laien, die auf diese Weise zustandekamen, stellt die Güteverhandlung vor dem Arbeitsgericht "Gegen Gotteslohn" dar: ${ }^{14}$ Über Zweidrittel der gesamten Verhandlung hinweg beeinflußt das idiosynkratische Verständnis des Ausdrucks "Güteverhandlung” des Klägers, das lange Zeit latent bleibt, die Handlungskoordination zwischen den Beteiligten; erst zu einem sehr späten Zeitpunkt wird diese Wahrnehmungsdivergenz eher zufällig offenbar und durch den Richter korrigiert; ich zitiere den Transkriptionsausschnitt mit der entsprechenden Reparaturaktivität des Richters (C) gegenüber dem Kläger (A2) und der Klägerin (A1):

C ja warum warum sin se dann net woanders hingegangen wenn ihnen

C des jetz so weh tut also ich versteh das nicht $\downarrow *$ was wolln se

C denn da" wieder mit aufrechnen $\downarrow *$

A2 es geht doch äh ich hab de äh die einladung bekommen— - daß es zu

A2 einer güter*einigung kommt und güterabwägung $\downarrow$

$\mathrm{C}$ + richtig $\downarrow$

A2 +und die güter die wir äh da da muß ich doch meine güter ne"nnen $\downarrow$

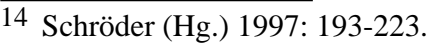


A1
C nein des heißt güte"verhandlung nicht güte"rverhandlung $\downarrow$
A2 ja mei $\mid$ ja da muß ich auch noch die dinge- $\downarrow$
C $\quad$ güte aber nich— $*$ das soll ne
gütliche einigung

15 A2 ja $\downarrow$

$\mathrm{C}$ das gut die einigung besteht darin daß jeder bissel nachgibt $\downarrow$ ne $\uparrow$

(Schröder (Hg.) 1997: 213)

Wie in der gesamten voraufgehenden Verhandlung diese Wahrnehmungsdivergenz, die durch die idiosynkratische Interpretation des Fachausdrucks "Güteverhandlung" ausgelöst wurde, die Verständigung und die Handlungskoordination beeinflußt und gestört hat, ist wieder bloßer Beobachtung nicht zugänglich, aus reiner Erinnerung, und sei diese durch Notizen gestützt, nicht erfaßbar oder rekonstruierbar. Nur die Tonaufnahme und das Transkript lassen das minuziöse Nachzeichnen solcher interaktiven Prozesse zu.

\subsection{Professionelles Interaktions- und Verfahrenswissen und das Orientierungswissen der Parteien als Konfliktpo- tential}

In Schröder 1997 habe ich dafür zu argumentieren versucht, daß man sich endlich konsequent von einem Fachsprachenkonzept lösen sollte, das Fachsprache als 'rein sprachlich-terminologisches' Phänomen handhabt, ob nun eher auf lexikalisch-semantischer, auf syntaktisch-stilistischer oder auf pragmatischer Ebene, und ich habe dafür plädiert, von "fachlicher Kommunikation" zu sprechen, weil eine erfolgreiche Beteiligung am fachlichen Diskurs kaum ein rein sprachliches Problem sei, sondern als genauso wichtig "das Wissen um institutionelle Konventionen oder Normen und die Vertrautheit und Erfahrung mit spezifischen Interaktionsregeln und Interaktionsmustern im jeweiligen professionellen Kontext" 15 einzuschätzen ist. Wie allein fehlendes Institutions- und Interaktionswissen auf der Seite des Laien und die Selbstverständlichkeit, mit der der Professionelle dieses Institutions- und Interaktionswissen als Handlungsressource benutzt, Turbulenzen in der Verhandlung auslösen kann, sie gefährden kann, will ich jetzt anhand der fragmentarischen Analyse zweier kleiner Gesprächsausschnitte aufzei-

15 Schröder 1997: 17f. 
gen, wieder aus der Güteverhandlung “Götz-Zitat” (vgl. oben). Wie Gerichtsverhandlungen laufen solche Güteverhandlungen vor dem Schiedsmann "nach spezifischen Interaktionsregeln ab, ihnen unterliegt ein Interaktions- oder Handlungsschema mit einer Reihe von charakteristischen Aktivitätskomplexen. Einer dieser Aktivitätskomplexe nach der 'Etablierung der Anschuldigung' ist im Rahmen der Rekonstruktion des Konflikts die 'Stellungnahme des Antragsgegners zum Anschuldigungssachverhalt':"16

\section{Rekonstruktion des Konflikts: Stellungnahme des Antragsgegners}

B äh \#sieben uhr fümunfünfzig bin ich auf den parkplatz

K KLINGT WIE ABGELESEN

25 B jegangen da ich regelmäßig um acht uhr einf| einkaufen fahre $\downarrow * \#$ $\mathrm{K}$

B \#und hab ich mein wagen gestartet und dabei stellt ich

K ERZÄHLTON

C ja:-

B fest daß die regen die: $w \mid$ schei”ben alle voller wasser warn—*

$\mathrm{K}$

01 B HOLT LUFT UND RÄUSPERT SICH und da bin ich au"sgestiegen hab $\mathrm{K}$

B mit=em leder die scheiben abputzen wollen $\uparrow \quad$ darauf \#kam\# frau

$\mathrm{K}$

SAGT KIAM

$\mathrm{C}$

$\mathrm{mhm}-$

B blank wutentbrannt auf=en par| auf den auf ihre veranda - *

$\mathrm{K}$

05 B und sachte mach den motor aus du umweltverschmutzer ich zeige

$\mathrm{K}$

B dich a"n $\downarrow *$ und da ist mir der kragen geplatzt $\downarrow \#$

K ENDE DES ERZÄHLTONS

$\mathrm{C}$

$>\mathrm{ja}-<* *$ und

16 Schröder 1997: 18; wie hier werde ich auch im folgenden teilweise die Analyse in Schröder 1997 direkt zitieren; vgl. auch wieder die ausführliche Analyse dieser Verhandlung in Schröder/Klein/Nothdurft/Reitemeier 1997. 
$\begin{array}{ll}\text { B da haben se diesen berühmten } & \begin{array}{l}\text { jawowohl> } \\ \text { ausdruck }\end{array} \text { von götz von berlichingen }\end{array}$

10

B jawohl-
C gesagt-

(Schröder (Hg.) 1997: 122f.)

"Diese für solche Güteverhandlungen typische Stellungnahme des Antragsgegners zum Anschuldigungssachverhalt, in dessen Verlauf Herr Herrmann mit allen rhetorischen Tricks die eigenen Schuldanteile zu Lasten der Antragstellerin Frau Blank zu minimieren bemüht ist, endet damit, daß er immerhin die inkriminierte Äußerung zugibt: und da ist mir der kragen geplatzt, eine Formulierung die ambivalent ist, da sie sowohl als metaphorische Umschreibung des Götz-Zitats (auf allerdings sehr allgemeiner Ebene) als auch als die bloße Wiedergabe der psychischen Disposition, aus der heraus Herr Herrmann dann schließlich 'deutlich' geworden ist, verstanden werden kann. Der Schiedsmann läßt ihm, wie der Gesprächsausschnitt zeigt, diese Vagheit nicht durchgehen und präzisiert selbst (und da haben se diesen berühmten ausdruck von götz von berlichingen gesagt $)^{17}$. Herr Herrmann bestätigt (jawohl) und hat damit - immer ein Schritt, der Überwindung kostet das eigentliche Delikt zugegeben. Das sollte eigentlich ausreichen, meint er wohl." (Schröder 1997: 19f.)

"Herr Herrmann irrt sich. Das Zugeben des Delikts an dieser Stelle der Verhandlung, in der es eher um eine gesprächsweise Klärung oder Rekonstruktion des Konflikts im Vorfeld einer rechtlich verbindlichen Regelung geht, ${ }^{18}$ erspart ihm nicht den formalen Akt des Zugebens im Zusammenhang mit einer rechtlich verbindlichen Konfliktregelung zu einem späteren Zeitpunkt in der Güteverhandlung. Dieser Situation sieht sich Herr Herrmann gegenüber, nachdem der Schiedsmann nun seinerseits noch einmal den Konfliktsachverhalt mit Hilfe juristischer Normen rekonstruiert und das Delikt strafrechtlich lokalisiert hat, ${ }^{19}$ und er reagiert deutlich ungehalten:" (Schröder 1997: 20)

$17 \mathrm{Zu}$ einer detaillierteren Rekonstruktion des Vorgangs vgl. Schröder/Klein/Nothdurft/Reitemeier 1997.

18 Von 'Unterhaltung darüber', wie das zustandegekommen ist, spricht der Schiedsmann, wenn er diese Phase der Güteverhandlung einleitend in ihrer Funktion markiert.

19 Vgl. die Analyse zur Terminologisierung im voraufgehenden Abschnitt. 
Regelung des Konflikts: Aushandlungsaktivitäten zum Einigungsvorschlag — Die Ratifizierung

$\mathrm{C}$ sie geben also zu das gesacht zu

B \#+hab ich eben ganz laut und deutlich gesacht $\downarrow \#$

$\mathrm{K} \quad$ KLINGT LEICHT VERÄRGERT

C haben- ja: gut ich frage

25

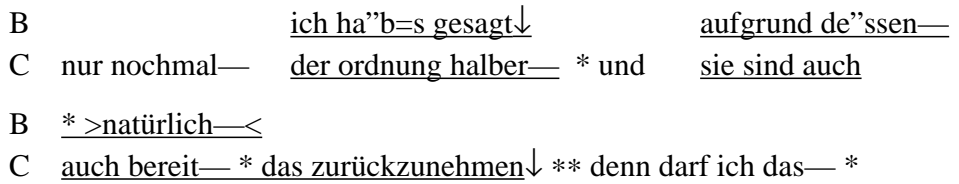

01

B $\quad$ so zu protokoll nehmen $\uparrow^{\mathrm{ja} \downarrow}+\mathrm{ja} \uparrow^{2}$

(Schröder (Hg.) 1997: 124)

Das eher gesprächsweise Zugeben des Delikts ist etwas anderes als das förmliche Schuldeingeständnis im Rahmen der rechtlich verbindlichen Konfliktregelung. Das war dem Antragsgegner nicht klar, für ihn ist das offensichtlich kein Unterschied, und er empfindet es deutlich als für ihn ärgerliches 'Vorgeführtwerden', zumal in Gegenwart der Streitgegnerin und ihrer Zeugin, seine Verfehlung in unnötiger Weise noch einmal zugeben zu müssen. Der Schiedsmann hat es in routinierter Abwicklung des Verfahrens unterlassen, den Parteien die Verfahrensrelevanz des Schrittes eines förmlichen Schuldeingeständnisses $\mathrm{zu}$ verdeutlichen und damit die Turbulenz letztlich ausgelöst. Nicht das fehlende terminologische Wissen des Laien, sondern sein fehlendes Institutions- und Interaktionswissen und die fehlende Sensibilität des Professionellen für die besonderen Interaktionsbedingungen und -anforderungen an die Beteiligten haben die Verständigung behindert und die Handlungskoordination erschwert. Wieder sei hinzugefügt: Aus Platzgründen habe ich ein relativ einfaches und 'durchsichtiges' Beispiel gewählt, um Orientierungsdivergenzen zwischen Professionellen und Laien sowie ihr verhandlungsgefährdendes Potential aufzuzeigen. Solche Orientierungsdivergenzen sind häufig wesentlich komplizierter und komplexer und treten nicht in der Weise in Erscheinung wie in dem hier vorgestellten Gesprächsausschnitt, sondern existieren als latente Einflußgrößen in der Verhandlung, etwa wenn, wie von einem Arbeitsrichter beobachtet, die klagenden Arbeitnehmer in den Arbeitsgerichtsverhandlungen in ihrer Wahrnehmung des Richters und des Verfahrens 
des öfteren vom frequenten Konsum amerikanischer Kriminalfilme beeinflußt sind und die entsprechenden Rechts- und Rechtsfindungskonzepte auf deutsche Verhältnisse übertragen.

\section{Schlußbemerkung}

Soweit der Versuch, meine Diskussionsbeiträge während des Workshops "Legal Texts" ein wenig auszubuchstabieren und auf ein etwas tragfähigeres Fundament zu stellen. Der Workshop war eigentlich schriftlichen Texten gewidmet; ich könnte mir aber vorstellen, daß die hier entwickelte dynamische und kommunikativ-funktionale Sicht auf juristische Kommunikation auch Anregungen enthält, die sich für eine Beschäftigung mit Texten produktiv nutzen ließen.

\section{Literatur}

Bergmann, Jörg R. (1981). Ethnomethodologische Konversationsanalyse. In Schröder \& Steger (Hg.) (1981). Dialogforschung. Jahrbuch 1980 des Instituts für deutsche Sprache. 9-51.

Bergmann, Jörg R. (1988). Ethnomethodologie und Konversationsanalyse. Kurseinheit 1 - 3. Hagen: Fernuniversität - Gesamthochschule. Fachbereich Erziehungs-, Sozialund Geisteswissenschaften.

Brinker, Klaus \& Sager, Sven F. (1989). Linguistische Gesprächsanalyse: Eine Einführung (= Grundlagen der Germanistik 30). Berlin: Erich Schmidt.

Brünner, Gisela \& Fiehler, Reinhard (1998). Linguistische Untersuchungen zur Wirtschaftskommunikation. In Sprachreport 3/1998. 13-16.

Caesar-Wolf, Beatrice \& Breunung, Leonie (1981). Zur Analyse von verbalen Interaktionsprozessen im gerichtlichen Verfahren am Beispiel einer Zeugenvernehmung im Zivilprozeß. In Ermert, Karl (Hg.) (1981). Sprache und Recht (= Loccumer Protokolle 31). Loccum: Evangelische Akademie Loccum. 17-66.

Deppermann, Arnulf (1997). Glaubwürdigkeit im Konflikt. Rhetorische Techniken in Streitgesprächen, Prozessanalysen von Schlichtungsgesprächen (= Europäische Hochschulschriften 184). Frankfurt: Peter Lang.

Fiehler, Reinhard (1999). Gesprächsforschung und Kommunikationstraining. In Textund Gesprächslinguistik 2: Gesprächslinguistik. Berlin: de Gruyter (HSK = Handbücher zur Sprach- und Kommunikationswissenschaft) (in Vorbereitung).

Goethes Werke (o.J.). Auswahl in zehn Teilen. Auf Grund der Hempelschen Ausgabe neu herausgegeben mit Einleitungen und Anmerkungen versehen von Karl Alt in Verbindung mit Robert Riemann und Eduard Scheidemantel. Berlin: Deutsches Verlagshaus Bong \& Co. (Goldene Klassiker-Bibliothek). 
Henne, Helmut \& Rehbock, Helmut (1979). Einführung in die Gesprächsanalyse. Berlin: de Gruyter.

Hoffmann, Ludger (1980a). Zur Pragmatik von Erzählformen vor Gericht. In Ehlich, Konrad (Hg.) (1980). Erzählen im Alltag. Frankfurt/Main: Suhrkamp. 28-63.

Hoffmann, Ludger (1980b). Sprechen vor Gericht. Ein Versuch zur Beschreibung von Kode-Merkmalen. In Zeitschrift für Semiotik 3/1980. 207-232.

Hoffmann, Ludger (1983). Kommunikation vor Gericht (= Kommunikation und Institution 9). Tübingen: Gunter Narr.

Hoffmann, Ludger (1989). Verstehensprobleme in der Strafverhandlung. In Hoffmann (Hg.) (1989). Rechtsdiskurse. 165-195.

Hoffmann, Ludger (Hg.) (1989). Rechtsdiskurse. Untersuchungen zur Kommunikation in Gerichtsverfahren (= Kommunikation und Institution 11). Tübingen: Gunter Narr.

Kallmeyer, Werner \& Schütze, Fritz (1976). Konversationsanalyse. In Studium Linguistik 1. 1-28.

Kallmeyer, Werner (Hg.) (1996). Gesprächsrhetorik. Rhetorische Verfahren im Gesprächsprozeß (= Studien zur deutschen Sprache 4). Tübingen: Gunter Narr.

Klein, Wolfgang (1987). Die Kunst des Schlichtens. Techniken der Herbeiführung von Einigung in einer Güteverhandlung vor dem Schiedsmann. In Röhl (Hg.) (1987). Das Güteverfahren vor dem Schiedsmann. 611-639.

Klein, Wolfgang (1995). Schlichten in der Vergleichsbehörde. Die Herbeiführung von Einigung. In Nothdurft (Hg.) (1995). Streit schlichten. 27-133.

Klein, Wolfgang \& Nothdurft, Werner (1987). "Die Rauferei" oder "dat andere thema". Einführende Bemerkungen zur Gesprächsanalyse einer Güteverhandlung vor dem Schiedsmann. In Röhl (Hg.) (1987). Das Güteverfahren vor dem Schiedsmann. 543564.

Nothdurft, Werner (1986). Zündstoff. Das Management explosiver Sachverhalte in Schlichtungsgesprächen. In Worstbrock, Franz Josef \& Koopmann, Helmut (Hg.) (1986). Formen und Formgeschichte des Streitens. - Der Literaturstreit. Bd. 2 der Akten des VII. Internationalen Germanisten-Kongresses, Göttingen 1985. Tübingen: Niemeyer. 12-33.

Nothdurft, Werner (1987). Die Ordnung des Konflikts. Gesprächsanalyse der Konfliktbehandlung. In Röhl (Hg.) (1987). Das Güteverfahren vor dem Schiedsmann. 565609.

Nothdurft, Werner (1989). Interaktive Paradoxa konsensueller Konfliktlösung. Der Fall des 'Schiedsmann'. In Hoffmann (Hg.) (1989). Rechtsdiskurse. 97-215.

Nothdurft, Werner (1995). Gesprächsanalyse von Schlichtung. Die Geschichte eines Forschungsprojekts und die Entwicklung seiner Ergebnisse. In Nothdurft (Hg.) (1995). Streit schlichten. 1-26. 
Nothdurft, Werner (Hg.) (1995). Streit schlichten. Gesprächsanalytische Untersuchungen zu institutionellen Formen konsensueller Konfliktregelung (= Schriften des Instituts für deutsche Sprache 5.1). Berlin: de Gruyter.

Nothdurft, Werner (1997). Konfliktstoff. Gesprächsanalyse der Konfliktbearbeitung in Schlichtungsgesprächen (= Schriften des Instituts für deutsche Sprache 5.2). Berlin: de Gruyter.

Rehbein, Jochen (1989). Mündliche Schriftlichkeit. Version einer Körperverletzung in einer Berufungsverhandlung. In Hoffmann (Hg.) (1989). Rechtsdiskurse. 251-326.

Reitemeier, Ulrich (1985). Studien zur juristischen Kommunikation. Eine kommentierte Bibliographie (= Forschungsberichte des Instituts für deutsche Sprache 56). Tübingen: Gunter Narr.

Reitemeier, Ulrich (1987). Subjektive Normorientierung und ihre Geltendmachung in einer Güteverhandlung vor dem Schiedsmann. Darstellungsverfahren des Konfliktund Rechtsverständnisses der beschuldigten Partei. In Röhl (Hg.) (1987). Das Güteverfahren vor dem Schiedsmann. 641-665.

Rottleuthner, Hubert (1978). Probleme der Beobachtung von Gerichtsverhandlungen, dargestellt am Beispiel richterlicher Vergleichstrategien und kompensatorischen Verhaltens im Arbeitsgerichtsverfahren. In Hassemer, Winfried \& Hoffman-Riem, Wolfgang \& Weiss, Manfred (Hg.) (1978). Interaktion vor Gericht (= Schriften der Vereinigung für Rechtssoziologie 2). Baden-Baden: Nomos. 109-132.

Rottleuthner, Hubert (Hg.) (1984). Rechtssoziologische Studien zur Arbeitsgerichtsbarkeit (= Schriften der Vereinigung für Rechtssoziologie 9). Baden-Baden: Nomos.

Röhl, Klaus F. (Hg.) (1987). Das Güteverfahren vor dem Schiedsmann. Soziologische und kommunikationswissenschaftliche Untersuchungen (Fachbücher für den Schiedsmann). Köln: Carl Heymanns Verlag.

Schröder, Peter (1987). Sprachliches Funktionieren und interaktive Funktion von Stereotypen in einer Güteverhandlung vor dem Schiedsmann. In Röhl (Hg.) (1987). Das Güteverfahren vor dem Schiedsmann. 667-722.

Schröder, Peter (1995). Besser ein magerer Vergleich als ein fetter Prozeß. Zur Güteverhandlung im Arbeitsgericht. In Nothdurft (Hg.) (1995). Streit schlichten. 134-267.

Schröder, Peter (1997). Zwischenruf. Plädoyer für eine eigentlich ganz selbstverständliche Perspektive für die Beschäftigung mit Fachsprache. In Hva er fagspråk? Was ist Fachsprache? What is LSP? 1 (= SPRÅK og MARKED 17). Halden. 17-22.

Schröder, Peter (Hg.) (1997). Schlichtungsgespräche. Ein Textband mit einer exemplarischen Analyse (= Schriften des Instituts für deutsche Sprache 5.3). Berlin: de Gruyter.

Schröder, Peter \& Klein, Wolfgang \& Nothdurft, Werner \& Reitemeier, Ulrich (1997). Schlichtungsgespräche verstehen. Exemplarische Fallanalyse einer Güteverhandlung vor dem Schiedsmann. In Schröder (Hg.) (1997). Schlichtungsgespräche. 1652. 
Schröder, Peter \& Steger, Hugo (Hg.). Dialogforschung. Jahrbuch 1980 des Instituts für deutsche Sprache (= Sprache der Gegenwart 54). Düsseldorf: Pädagogischer Verlag Schwann.

Spranz-Fogasy, Thomas (1986). 'Widersprechen'. Zu Form und Funktion eines Aktivitätstyps in Schlichtungsgesprächen. Eine gesprächsanalytische Untersuchung (= Forschungsberichte des Instituts für deutsche Sprache 62). Tübingen: Gunter Narr.

Ullmer-Ehrich, Veronika (1981). Linguistische Aspekte der forensischen Argumentation. In Schröder \& Steger (Hg.) (1981). Dialogforschung. 188-225.

Wolff, Stephan \& Müller, Hermann (1997). Kompetente Skepsis. Eine konversationsanalytische Untersuchung zur Glaubwürdigkeit in Strafverfahren. Opladen: Westdeutscher Verlag.

\section{Legende der verwendeten Transkriptionszeichen}

$\mathrm{B}=$ Antragsgegner (Beklagter)

$\mathrm{C}=$ Schiedsmann

$\mathrm{K} \quad=$ Kommentarzeile

Hier erscheinen

- verbale Wiedergaben von Aktionalem durch den Transkribenten, und zwar sowohl nichtkommunikative Ereignisse, die zur Situation gehören, als auch solche, die von außen eindringen

- verbale Wiedergaben von kollektiven non-verbalen Handlungen oder von nonverbalen Handlungen eines Sprechers, die er vollzieht, während er spricht

- Interpretationen des Transkribenten von Äußerungen der Sprecher / Wiedergabe von atmosphärischen Eindrücken wie FEUNDLICHER TON / ENERGISCH / ENTSCHULDIGEND etc.

- Informationen des Trankribenten zur Aufnahme und Situation.

\# \# markiert auf der bzw. den Sprecherzeilen den Bereich der Gültigkeit eines

$\downarrow \quad$ Kommentars

$\downarrow \quad$ fallende Intonation (z.B. "jetzt stimmt es(")

$\uparrow \quad$ steigende Intonation (z.B. "kommst du mit(")

- $\quad$ schwebende Intonation (z.B. "ich sehe hier-")

* $\quad$ kurze Pause

** etwas längere Pause (bis max. 1 Sekunde)

| Wortabbruch

: $\quad$ auffällige Dehnung (z.B. "ich war so: fertig”)

“ auffällige Betonung (z.B. “aber ge”rn”)

$=\quad$ Verschleifung (Elision) eines oder mehrerer Laute zwischen Wörtern (z.B. "sa=mer" für "sagen wir") 
$>\quad$ leiser (relativ zum Kontext)

$<$ lauter (relativ zum Kontext)

ja aber simultan gesprochene Äußerungen stehen übereinander nein und sind unterstrichen

$+\quad$ unmittelbarer Anschluß (Anklebung) bei Sprecherwechsel 